\title{
Manifestações Bucais de Pacientes HIV Atendidos na Fundação de Medicina Tropical do Amazonas (FMT-AM)
}

Oral Manifestation in HIV Positive Patients Attended at Amazonas Tropical Medicine Foundation (FMT-AM)

\section{Michelle Valle das Chagas ${ }^{1}$, Lucilaide Oliveira Santos ${ }^{2}$, Lia Mizobe Ono ${ }^{3}$}

\section{Abstract}

One hundred HIV positive patients were evaluated using a descriptive observational cohort study at the Foundation of Tropical Medicine in the State of Amazon (FMT-AM), from December 2007 to April 2008, in order to assess the occurrence of oral manifestations. Seventy patients were male (average age was 36.78 years) and thirty females (average age was 32.36 years). The most frequent oral manifestations of HIV were: oral candidiasis $(54 \%)$ in its clinical types - pseudomembranous, eritematous and angular queilitis; gingivitis (25\%); periodontitis (18\%); recurrent aphthous stomatitis (7\%); hairy leukoplakia (5\%); Kaposi's sarcoma $(5 \%)$; cytomegalovirus (3\%); herpes simplex $(2 \%)$; Herpes Zoster $(2 \%)$. Only $13 \%$ patients had no oral manifestations. These results indicate the importance of the knowledge on the most common oral lesions found in HIV positive patients and the importance of a thorough oral clinical examination in the course of HIV infection for early diagnosis and adequate treatment.

Keywords: HIV infection; AIDS; Oral pathologies; Oral candidiasis.

\section{Resumo}

Foram analisados através de um estudo de coorte observacional descritivo, 100 pacientes HIV/AIDS na Fundação de Medicina Tropical do Amazonas (FMT-AM) no período de dezembro de 2007 a abril de 2008 com o objetivo de identificar as manifestações bucais desta doença. Setenta pacientes eram do gênero masculino, com média de idade de 36,78 anos, e trinta do gênero feminino, com idade média de 32,36 anos. As manifestações bucais mais freqüentes foram a candidíase $(54 \%)$, nas formas pseudomembranosa, eritematosa e queilite angular, a gengivite (25\%), a periodontite (18\%), a estomatite aftosa recorrente $(7 \%)$, a leucoplasia pilosa (5\%), o sarcoma de Kaposi (5\%), a infecção pelo citomegalovírus $(3 \%)$, o herpes simples $(2 \%)$ e o herpes zoster (2\%). Apenas $13 \%$ dos pacientes não apresentaram qualquer manifestação bucal. Esses dados apresentados mostram a importância do conhecimento das lesões mais comumente encontradas no paciente HIV positivo, do exame clínico bucal minucioso para o diagnóstico precoce e assim poder planejar um tratamento bucal adequado.

Palavras-chave: Infecção pelo HIV; AIDS; Patologias bucais; Candidíase bucal.
${ }^{1}$ Aluna do curso de Odontologia da Universidade do Estado do Amazonas (UEA)

${ }^{2}$ Médica da Fundação de Medicina Tropical do Amazonas (FMT-AM), Mestre em Medicina Tropical- FIOCRUZ

${ }^{3}$ Professora de Patologia Bucal da Universidade do Estado do Amazonas (UEA), Doutoranda em Clinica Odontológica- UNICAMP

Correspondência: Michelle Valle das Chagas

Endereço: Rua ON, 07 casa 04 - CEP 69060-068, Manaus - AM, Brasil

E-mail: lono@uea.edu.br

Data de Submissão: 15/11/2009

Data de Aceite: 03/10/2010

\section{Introdução}

A Síndrome da Imunodeficiência Adquirida, conhecida mundialmente como AIDS é a manifestação clínica avançada da infecção pelo vírus da imunodeficiência humana (HIV), descrita pela primeira vez em 1981, nos Estados Unidos, em pacientes homens, homossexuais, que tinham em comum uma significante diminuição da resposta imunológica. Desde então, há um aumento contínuo e progressivo do número de pacientes infectados, com aproximadamente 33.2 milhões de pacientes em 2007 (MATTOS; SANTOS; FERREIRA, 2004; PEDREIRA et al., 2008; UNAIDS, 2007).

A AIDS é caracterizada por uma severa imunossupressão do hospedeiro, manifestando-se por uma grande variedade de sintomas e sinais clínicos, sendo muitos observados na boca. As manifestações bucais geralmente se apresentam no curso da doença e são facilmente identificadas e diagnosticadas (CAVASSANI et al., 2002; COOGAN et al., 2005; REZNIK,2005/2006; ZHANG; REICHART; SONG, 2009).

Alguns pesquisadores dividiram as manifestações associadas à infecção pelo HIV em três grupos, segundo o qual os grupos I e II representam as lesões de ocorrência na região de cabeça e pescoço, mais e menos freqüentes respectivamente, já o grupo III abrange lesões possivelmente associadas com infecção pelo HIV. Verifica-se na literatura que as manifestações bucais de modo geral, correspondem a aquelas por infecções fúngicas, bacterianas e virais. Dentre as infecções fúngicas, a candidíase bucal pelo Candida albicans, é uma das doenças oportunistas mais comuns associadas à infecção pelo vírus HIV, sendo as formas eritematosa e pseudomembranosa as mais incidentes, seguidas da queilite angular. Acredita-se que estas manifestações estejam relacionadas à imunossupressão causada pela diminuição dos linfócitos CD4 e a fatores locais, como a xerostomia (AXELL; BAERT; BROCHERIOU, 1991; CAVASSANI et al., 2002; ERKOSE; ERTURAN, 2007; NARANI; EPSTEIN, 2001; REZNIK, 2006; SHARMA et al., 2009; SOUZA et al., 2000; YENGOPAL; NAIDOO, 2008).

A leucoplasia pilosa é infecção pelo vírus Epstein-Barr, comumente encontrada em pacientes nos estágios iniciais da AIDS, sendo o bordo lingual a região mais afetada e manifestandose clinicamente como uma placa branca de superfície plana, corrugada ou pilosa. O herpes simples, causado pelo vírus HSV-1, apresenta-se como vesículas pequenas, dolorosas que podem coalescer e formar ulcerações maiores que ao persistir por mais de duas semanas associadas à soropositividade para HIV, é 
conclusivo para um diagnóstico de AIDS. Outras infecções virais que podem comprometer o paciente imunossuprimido são o herpes zoster, causado pelo vírus varicela-zoster (VVZ), o condiloma acuminado causado pelo HPV e a infecção pelo citomegalovírus (CMV) (DIAS et al., 2001; FARIA et al., 2005; KENNEDY; GRIFELD; GOW, 1998; MOURA et al., 2005; RAFAILIDIS et al., 2008; REZNIK, 2005/2006). O herpesvirus humano-8 (HHV-8) está fortemente relacionado ao sarcoma de Kaposi (SK) e pode ser facilmente detectado por biópsia nos tecidos infectados ou por exame do sangue periférico. As fases iniciais são caracterizadas por manchas roxas, que evoluem para placas e posteriormente apresenta-se como nódulo tumoral (NSUBUGA et al., 2008 RAMOS-DA-SILVA, 2006).

Dentre as infecções bacterianas destaca-se a gengivite associada ao HIV, caracterizada por severo eritema da gengiva marginal, inserida e mucosa alveolar. Pode apresentar um halo eritematoso e pode ou não ser acompanhada de ocasionais hemorragias e desconforto. Outra descrita é a periodontite associada à necrose que pode levar os dentes à mobilidade devido a rápida perda do tecido ósseo e mole, resultar em hemorragias, odor desagradável e dor intensa por toda maxila e mandíbula. Existem hipóteses de que o Epstein-Barr vírus esteja envolvido na evolução da doença periodontal (IMANISHI, 2005). Outras manifestações de interesse são as úlceras atípicas de etiologia desconhecida, podendo apresentar-se com diferentes graus de severidade (REZNIK, 2005/2006).

A introdução da terapia anti-retroviral potente, composta pela combinação de três ou mais drogas, em substituição à monoterapia, constituiu um grande avanço na luta contra a infecção pelo HIV. Com a redução da morbidade, mortalidade e melhoria na qualidade de vida, a procura dos pacientes HIV-positivos por cuidados bucais também está aumentando. Independentemente da gravidade, natureza infecciosa e estigma social criado pela doença, qualquer profissional de saúde deve estar apto a dar um bom atendimento a esses pacientes, o que implica na necessidade de deter conhecimentos sobre as manifestações bucais associadas ao HIV e sua relação com a progressão da infecção (MATTOS SANTOS; FERREIRA, 2004). Sendo assim, este estudo teve como objetivo identificar as principais manifestações bucais em pacientes portadores de HIV/AIDS atendidos na Fundação de Medicina Tropical do Amazonas (FMT-AM).

\section{Metodologia}

Foi realizado um estudo de coorte observacional descritivo, onde foram examinados 100 pacientes internados no setor de AIDS da FMT-AM, no período compreendido entre dezembro de 2007 a abril de 2008, assim foram estabelecidos os seguintes métodos de inclusão para o estudo: pacientes internados no período de dezembro de 2007 a abril de 2008 portadores do HIV. O critério de exclusão estabelecido foram os pacientes internados na Unidade de Terapia Intensiva (UTI).

A pesquisa realizada teve aprovação do Comitê de Ética em Pesquisa da FMT-AM (protocolo número 2806-07).

Os pacientes foram examinados por uma única examinadora que após constatar a soropositividade dos pacientes através dos resultados do Western Blot, realizou o exame intrabucal para verificar a presença de lesões e coletou os dados dos prontuários referentes ao gênero, a faixa etária e ocorrência de doenças sistêmicas associadas ao HIV foram obtidos dos prontuários. Os dados foram tabulados e apresentados como percentuais na forma de gráficos, preparados no software Microsoft 2003

\section{Resultados}

Dos 100 pacientes examinados (idades variando entre 17 a 68 anos), 70 eram do gênero masculino (média 36,78 anos) e 30 do feminino (média 32,36 anos) (Gráfico 1). Em relação à presença de manifestações bucais nesses pacientes, verificou-se a ocorrência de candidíase (54\%), leucoplasia pilosa (5\%), sarcoma de Kaposi (5\%), herpes simples (2\%), herpes zoster $(2 \%)$, gengivite $(25 \%)$, periodontite (18\%), citomegalovírus (3\%) e estomatite aftosa recorrente (7\%). Alguns pacientes não apresentaram manifestações bucais (13\%). (Gráfico 2).

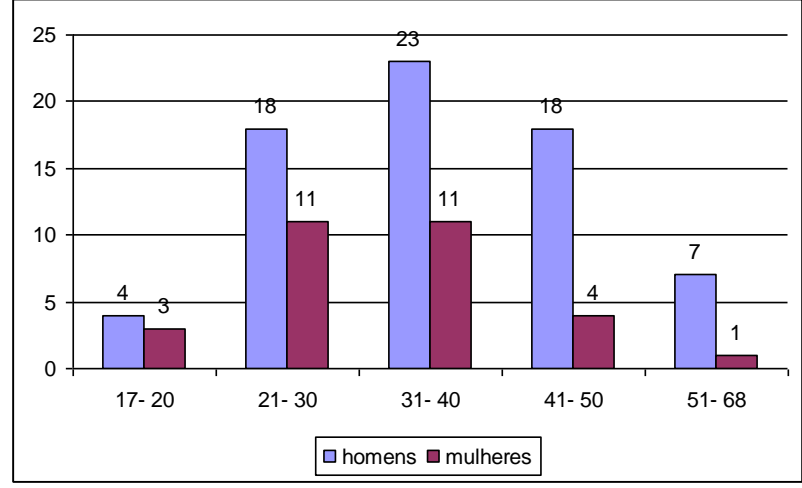

Figura 1. Distribuição dos HIV positivos com manifestações bucais de acordo com a faixa etária.

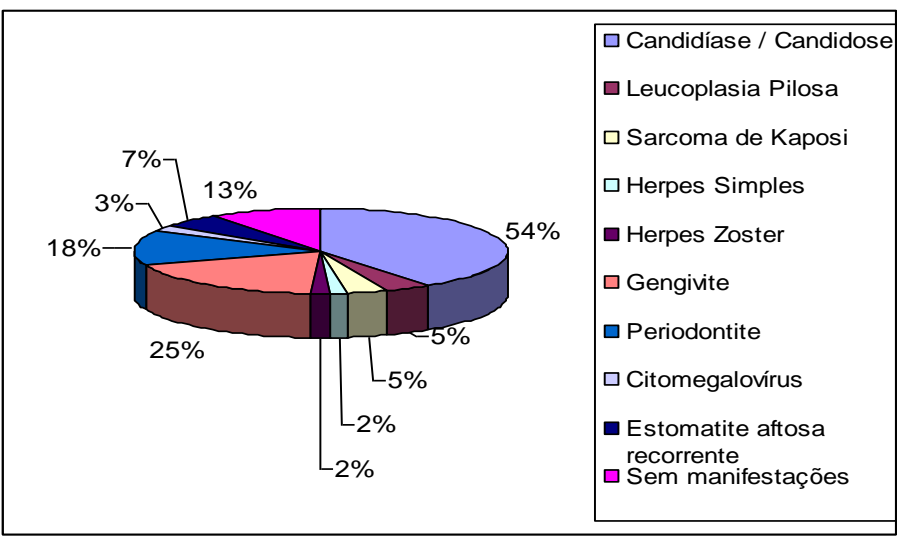

Figura 2. Manifestações bucais observadas nos pacientes HIV positivos.

Quando analisamos a distribuição das lesões de candidíase nas diversas apresentações clínicas, verificamos que a forma pseudomembranosa esteve presente em 32 pacientes do gênero masculino e 6 do feminino, seguida da quelite angular com 13 casos em pacientes do gênero masculino e 4 do feminino. A forma eritematosa foi constatada em 5 pacientes do gênero masculino e 7 do feminino. Apenas 6 pacientes do gênero masculino e 7 do feminino não apresentaram manifestações bucais.

Quanto à localização anatômica, foi observado que as lesões por candidíase estiveram presentes no dorso lateral da língua, mucosa jugal e labial, palato duro e mole; os casos de sarcoma de Kaposi ocorreram somente no palato duro; a infecção pelo citomegalovírus, a estomatite aftosa recorrente na mucosa jugal; o herpes simples na mucosa jugal e labial; o herpes zoster estiveram presentes na face e mucosa jugal e os casos de leucoplasia pilosa ocorreram no borda lateral da língua.

Dentre as manifestações sistêmicas destacaram-se as síndromes diarréicas, neurológicas e respiratórias, tais como a tuberculose (pulmonar, ganglionar, pleural), a neurotuberculose, a toxoplasmose, a neurocriptococose e a dermatofitose. A diarréia crônica e a pneumonia foram as manifestações mais observadas. Sepse e insuficiência respiratória foram algumas das maiores causas de óbitos de alguns desses pacientes. 


\section{Discussão e Conclusão}

Em nosso estudo verificamos que 83\% apresentaram manifestações bucais como mostram outros trabalhos (BRAVO et al., 2006; SHARMA et al., 2009). Houve predominância de pacientes do gênero masculino (70\%) sobre o feminino (30\%). Esses achados são semelhantes aos encontrados por outros autores (BENDICK; SCHEIFELE; REICHART, 2002; EYESON et al., 2002; NITTAYANANTA et al., 2002; PEDREIRA et al., 2008; SHARMA et al., 2006; SOUZA et al., 2000). Entretanto, os mesmos diferem do que foi observado em estudos anteriores realizados no Zimbábue (CHIDZONGA, 2003), onde a relação entre pacientes masculinos: femininos foi de $1: 1$ na África do Sul, onde a predominância foi do gênero feminino (YENGOPAL; NAIDOO, 2008).

Diversos trabalhos enfocaram nas manifestações bucais de pacientes HIV, mostrando a predominância de alguns tipos de lesões como a candidíase nas suas diversas formas clínicas, as doenças gengivais e periodontais, a leucoplasia pilosa, o sarcoma de Kaposi e o herpes simples (PEDREIRA et al., 2008; SOUZA et al., 2000), Estes achados estão de acordo com os observados no presente estudo. No entanto, um estudo realizado no Brasil não relatou casos de sarcoma de Kaposi dentre as manifestações bucais em pacientes HIV positivos (GASPARIN et al., 2009).

$A$ incidência das formas da candidíase encontradas em nosso trabalho estão de acordo com os achados de Souza et al. (2000), onde em ordem decrescente, encontram-se a forma pseudomembranosa, a queilite angular e a eritematosa. Por outro lado, Pedreira et al (2008) encontraram a forma eritematosa como a mais comum, seguida da pseudomembranosa e da queilite angular (PEDREIRA et al., 2008). Independente da forma clínica, essa infecção fúngica tem sido amplamente associada com a infecção pelo HIV, estando presente em todos os artigos revisados sobre prevalência de manifestações bucais em pacientes HIV positivos, sendo a lesão mais comum nos estudos realizados em várias partes do mundo (REZNIK, 2005/2006).

A leucoplasia pilosa foi observada em $5 \%$ dos nossos pacientes e quando comparamos a vários estudos, notamos uma marcante variedade entre os achados, uma vez que estes corresponderam a $1,3 \%$ dos casos no Zimbábue (CHIDZONGA, 2003), a 9,9\% dos casos na Inglaterra (EYESON et al., 2002), a $(15,8 \%$ dos casos na Índia) ( SHARMA et al., 2009), a $26 \%$ dos casos na Tailândia ( NITTAYANANTA et al., 2002), 35,5\% no Camboja (BENDICK et al., 2002) e 45,5\% dos casos no Brasil (FARIA et al., 2005). Quando examinados os pacientes HIV com tuberculose, encontramos a leucoplasia pilosa em $66,67 \%$ dos pacientes. A associação entre leucoplasia pilosa e a candidíase pseudomembranosa ou a leucoplasia pilosa e a pneumonia já havia sido relatada anteriormente (BENDICK; SCHEIFELE; REICHART, 2002).

A infecção pelo herpes simples no lábio inferior fol encontrada em $2 \%$ dos pacientes. Em estudos realizados na Nigéria (ADUROGBANGBA et al., 2004) e na Índia (SHARMA et al., 2006) essa infecção foi menos prevalente, correspondendo a 0,9\% dos pacientes, respectivamente. A prevalência no Camboja foi um pouco maior com 7,9\% (BENDICK; SCHEIFELE; REICHART, 2002).

Em indivíduos HIV-positivos observa-se ulcerações na mucosa bucal que estão relacionadas ao estado imunitário do paciente (MIZIARA; ARAÚJO FILHO; WEBER, 2005). Úlceras bucais são relatadas vários estudos em HIV-positivos, sendo que existem variações na prevalência desta como visto na India (3,8\%, SHARMA et al., 2009), na China (14,8\%, ZHANG et al., 2009) e na Inglaterra (23, EYESON et al., 2002). No presente estudo, $7 \%$ dos pacientes apresentaram esta lesão bucal, mostrando a variabilidade de expressão desta lesão.

A doença periodontal tem sido previamente associada à infecção pelo HIV, a qual é considerada um modificador da doença periodontal (VASTARDIS et al., 2003). Podemos observar diferentes incidências como visto em um estudo realizado na
Nigéria onde apresentou 4,3\% de casos de gengivite e 2,1\% de periodontite associada ao HIV (ADUROGBANGBA et al., 2004). Em contraste, no presente estudo observou-se que $25 \%$ dos pacientes apresentaram gengivite e $18 \%$, periodontite.

A análise dos resultados nos permite concluir que $83 \%$ dos pacientes apresentavam alguma manifestação bucal e $60 \%$ dos pacientes apresentavam mais de uma lesão. As mais freqüentes foram a candidíase pseudomembranosa e as doenças periodontais. $O$ sexo masculino foi $O$ mais acometido pelas manifestações bucais e pela doença HIV/AIDS com $70 \%$ dos casos. Os dados apresentados mostram a importância do conhecimento das lesões mais comumente encontradas no paciente HIV positivo, do exame clínico bucal minucioso para o diagnóstico precoce e assim poder planejar um tratamento bucal adequado. Sendo assim, se fazem necessários programas de orientação aos profissionais da área de saúde e de prevenção para a população em geral.

\section{Agradecimentos}

Ao Programa de Fomento à Iniciação Científica PROFIC/PAIC/FAPEAM, pelo apoio financeiro e à Fundação de Medicina Tropical do Amazonas (FMT-AM) que viabilizou a realização deste projeto.

\section{Referências}

ADUROGBANGBA, M.I. et al. Oro-facial lesions and CD4 counts associated with HIV/AIDS in an adult population in Oyo State, Nigeria. Oral Dis., Houndmills, v. 10, no. 6, p. 319-326, Nov. 2004.

AXELL, T.; BAERT, S.; BROCHERIOU, C. Revised classification of HIV - associated oral lesions. Br. Dent. J., London, v. 170, no. 8, p. 305-306, Apr. 1991.

BENDICK, C.; SCHEIFELE, C., REICHART, P.A. Oral manifestations in 101 Cambodian patients with HIV infection and AIDS. J. Oral Pathol. Med., Copenhagen, v. 31, no. 1, p.1-4, Jan. 2002.

BRAVO, I.M et al. H. Prevalence of oral lesions in HIV positive related to CD4 cell count and viral load in a Venezuelan population. Med. Oral Patol. Oral Cir. Bucal, Valencia, v. 11, no. 1, p. E33E39, jan. 2006.

CAVASSANI, V.G.S. et al. Candidíase oral como marcador de prognóstico em pacientes portadores do HIV. R. Bras. Otorrinolaringol., Rio de Janeiro, v. 68 , n. 5, p. 630-634, set./out. 2002.

CHIDZONGA, M.M. HIV/AIDS orofacial lesions in 156 Zimbabwean patients at referral oral and maxillofacial surgical clinics. Oral Dis. Houdmills, UK, v. 9, no. 6, p. 317-322, Nov. 2003.

COOGAN, M.M. et al. Oral lesions in infection with human immunodeficiency virus. Bull. World Health Org., Geneva, v. 83 no. 9, p. 700-706, Sept. 2005.

DIAS, E.P. et al. Leucoplasia pilosa oral: aspectos histopatológicos da fase subclínica. Pesq. Odontol. Bras., São Paulo, v. 15, n. 2, p. 104-111, abr./jun. 2001.

ERKOSE, G.; ERTURAN, Z. Oral Candida colonization of human immunodeficiency virus infected subjects in Turkey and its relation with viral load and CD4+ T-lymphocyte count. Mycoses, Berlin, v. 50 , no. 6 , p. $485-490$, Nov. 2007.

EYESON, J.D. et al. Oral manifestations of an HIV positive cohort in the era of highly active anti-retroviral therapy (HAART) in South 
London. J. Oral Pathol. Med., Copenhagen, v. 31, no. 3, p.169174, Mar. 2002

FARIA, P.R. et al. Tongue disease in advanced AIDS. Oral Dis., Houdmills, UK, v. 11, no. 2, p. 72-80, Mar. 2005.

GASPARIN, A.B. et al. Fatores e prevalências associados às manifestações bucais em pacientes HIV positivos atendidos em cidades sul-brasileiras. Cad. Saúde Pública, Rio de Janeiro, v. 25 , n. 6 , p. $1307-1315$, jun. 2009

IMANISHI, S.A. W. A investigação do citomegalovírus e do Epstein-Barr vírus na doença periodontal humana. 2005. $96 \mathrm{f}$ Dissertação (Mestrado em Odontologia) - Faculdade de Odontologia, Universidade Federal de Minas Gerais, Belo Horizonte.

KENNEDY, P.G.E.; GRIFELD, E.; GOW, J.W. Latent varicellazoster virus is located predominantly in neurons in human trigeminal ganglia. Proc. Natl. Acad. Sci., Washington, v. 95, no. 8, p. 46584662, Apr. 1998.

MATTOS, S.L.; SANTOS, V.R.; FERREIRA, E.F. Prevalência de lesões de mucosa bucal em pacientes HIV positivos de Unidade de Referencia Especializada em Doenças Infecciosas e Parasitarias Especiais -URE- DIPE (Belém-Pará). R. Bras. Patol. Oral, Natal v. 3, n. 1, p. 7-16, jan./mar. 2004

MIZIARA, I.D.; ARAÚJO FILHO, B.C.; WEBER, R. Aids e estomatite aftóide recidivante. R. Bras. Otorrinolaringol., Rio de Janeiro, v. 71, n. 4, p. 517-520, jul./ago. 2005

MOURA, M.D.G. et al. Condiloma acuminado na mucosa bucal de pacientes HIV positivos: relato de três casos clínicos. RPG: R. PósGrad. Fac. Odontol. Univ. São Paulo, São Paulo, v. 12, n. 4, p. 489- 491, out./dez. 2005

NARANI, N.; EPSTEIN J.B. Classifications of oral lesions in HIV infection. J. Clin. Periodontol., Copenhagen, v. 28, no. 2, p. 137145, Feb. 2001

NITTAYANANTA, W. et al. Co-existence between oral lesions and opportunistic systemic diseases among HIV-infected subjects in Thailand. J. Oral Pathol. Med., v. 31, no. 3, p. 163-168, Mar. 2002.

NSUBUGA, M.M. et al. Human herpesvirus 8 load and progression of AIDS-related Kaposi sarcoma lesions. Cancer Lett., Amsterdam, v. 263 , no. 2 , p. $182-188$, May 2008.

PEDREIRA, E.N. et al. Epidemiological and oral manifestations of HIV positive patients in a specialized service in Brazil. J. Appl. Oral Sci., Bauru, v. 16, no. 6, p. 369-3 75, Nov./Dec. 2008.

RAFAILIDIS, P. I. et al. Severe cytomegalovirus infection in apparently immunocompetent patients: a systematic review. Virol. J., London, v. 5, p. 47, Mar. 2008.

REZNIK, D. A. Oral manifestations of HIV disease. Top. HIV Med. San Francisco, CA, v. 13, no. 5, p. 143-148, Dec. 2005/Jan. 2006.

SHARMA, G. et al. Oral manifestations in HIV/AIDS infected patients from Índia. Oral Dis., Houdmills, v. 12, no. 6, p. 537-542, Nov. 2006

SHARMA, G. et al. Oral manifestations as predictors of immune suppression in a HIV-/AIDS-infected population in south India. Clin. Oral Invest., Berlin, v. 13, no. 2, p. 141-148, June 2009.
RAMOS-DA-SILVA, S. et al. Kaposi's sarcoma associated herpesvirus infection and Kaposi's sarcoma in Brazil. Braz. J. Med. Biol. Res., São Paulo, v. 39, no. 5, p. 573-580, May 2006.

SOUZA, L. B. et al. Manifestações orais em pacientes com AIDS em uma população brasileira. Pesq. Odontol. Bras., São Paulo, v. 14, n. 1, p. 79-85, jan./mar. 2000

UNAIDS. Global Summary of the AIDS Epidemic. Geneva: Joint United Nations Programme on HIV/AIDS (UNAIDS) and the World Health Organizations (WHO). Disponível em: < http://data.unaids.org/pub/EPISlides/2007/2007_epiupdate_en.pdf. dec. 2007 >. Acesso em: 2009.

VASTARDIS, S.A. et al. Periodontal disease in HIV-positive individuals: association of periodontal indices with stages of HIV disease. J. Periodontol., Chicago, v. 74, no. 9, p. 1336-1341, Sept. 2003

YENGOPAL, V.; NAIDOO, S. Do oral lesions associated with Hiv affect quality of life? Oral Surg. Oral Med. Oral Pathol. Oral Radiol. Endod., St. Louis, v. 106, no. 1, p. 66-73, July 2008.

ZHANG, X.; REICHART, P.A.; SONG, Y. Oral manifestations of HIV/AIDS in China: a review. Oral Maxillofac. Surg., Berlin, v. 13, no. 2, p. 63-68, June 2009. 